\title{
A Survey of Biosafety Practices in Clinical Laboratories Personnel from 12 Selected Areas of Karachi, Pakistan
}

\author{
Ahmad S, Ali B, Khan S, Fatima A, Saeed M, Asghar A, Akber SS*, Kazmi SU and Baqai R
}

Department of Clinical Microbiology and Immunology, Karachi, Dadabhoy Institute of Higher Education, Karachi, Pakistan

\begin{abstract}
The objective of this study was to realize the degree of biosafety precautions and measures recorded among different Private Hospitals and Diagnostic labs personnel situated in different areas of Karachi including SITE Area, Orangi Town, North Nazimabad, Nazimabad, New Karachi, Sadar, Malir Cant, Gulshan-iqbal, Gulistan-johar, Tariq road, Quaid Abad and F.B Area in Karachi, Pakistan. One thirty two private hospital based laboratory technicians were selected from all 12 selected areas in Karachi. The laboratory technicians were interviewed then a survey form was completed by the interviewer. The survey was conducted in 3 months duration from May 2017 to July 2017. The total number of members in this study was 132, of which 85 were males and 47 were females. Results presented that $65 \%$ of the laboratory technicians did not use any type of PPE, and $35 \%$ of the respondents recapped used syringes frequently while $25 \%$ recapped occasionally. For avoiding the reuse of syringes, Pakistani governments recommend that they be cut before discarding; however, only $65 \%$ of the respondents followed the given procedure. Even though mouth pipetting is considered outdated, $45 \%$ of the technicians continue to do so for numerous purposes. Moreover, standard operating procedures were not obtainable in $59.9 \%$ of the labs, and accidental records were not maintained in $72.5 \%$. Results of this survey established an absence of awareness about good and proper lab practices and lab biosafety measures amongst lab technicians in Karachi, Pakistan.
\end{abstract}

Keywords: Health and human service; Occupational health; Hospital

\section{Introduction}

Persons working in clinical diagnostic laboratories are exposed to many risks. The more the laboratorians become aware of and adhere to recommended, science-based safety precautions, the lower the risk for laboratory acquired infections [1]. In laboratory associated infections and biosafety, the most hazardous agent in the laboratory is a microorganism that is frequently associated with laboratory infections and can be transmitted in variety of routes, especially by aerosols [2]. In addition, a Knowledge, Attitude and Practice (KAP) study conducted in India revealed that numerous laboratory procedures and equipment are considered as a potential aerosol sources for health care workers unless standard infection control measures are implemented [3]. A study conducted on health care workers about the transfer of microorganisms from Personal Protective Equipment has shown that laboratory personnel are one of the risk groups among health care workers for occupational hazards [4]. They are exposed to variety of occupational health hazards including infectious materials, blood and other body fluids from patients including urine, pus, stool, secretion, sputum or equipment contaminated with agents in clinical laboratory atmosphere. In addition the behavior patterns and attitude of individuals towards safety programs influence their involvement in laboratory accidents that put themselves and fellow workers at risk. Personnel behavior towards laboratory safety plays vital role in the control and prevention of infections. For example carelessness, poor technique in the handling of infectious materials, needle sting or infectious aerosol exposures are the cause of laboratory acquired infections. Caring for patients with communicable diseases places health care workers including laboratorians at risk. Laboratory personnel in hospitals generally face with many hazards at work and their health and safety may be severely jeopardized unless adequate preventive protective measures are taken.

Biosafety is a concept that promotes safe and hygienic laboratory practices, measures and proper usage of containment equipment and facilities through laboratory staffs. A lot of accidents occur in the laboratory due to lack of proper knowledge regarding laboratory measures, indifferent attitude and improper implementation of safe laboratory practices [5]. Biosafety is an important issue in laboratory settings worldwide especially in developing countries where standard operating procedures are either lacking or not followed properly [6]. As in many other developing countries, biosafety is a critical issue that needs full attention to resolve in the health and diagnostic laboratory system of Pakistan. Laboratory person who handle blood or any biological sample can be at risk for accidental injury or exposure to contamination. Private and government sector hospital-based laboratory workers always contract with different pathogens; they are at risk for occupational infection [7]. Biosafety in lab work and the transporting of lab material from one place to another is a very critical tool in the worldwide fight against infectious diseases and exposures to laboratory personnel, particularly those working in microbiological laboratories as they are exposed to biohazards which may result in laboratory acquired infections [8]. Due to lack of proper awareness about biosafety issues causes the inappropriate handling and hazardous laboratory practices during collection of sample, its processing, specimens discarding etc are resulting laboratory technicians extra exposed to the pathogens. In Pakistan ignorance among laboratory technicians is mostly due to absence of awareness and the shortage

*Corresponding author: Syeda Sadaf Akber, Department of Clinical Microbiology and Immunology, Dadabhoy Institute of Higher Education, Karachi, Pakistan, Tel: 92 51-927-1617; E-mail: akbersadaf@gmail.com

Received October 18, 2018; Accepted December 04, 2018; Published December 14,2018

Citation: Ahmad S, Ali B, Khan S, Fatima A, Saeed M, et al. (2018) A Survey of Biosafety Practices in Clinical Laboratories Personnel from 12 Selected Areas of Karachi, Pakistan. Health Care Current Reviews 6: 234. doi: 10.4172/23754273.1000234

Copyright: @ 2018 Ahmad S, et al. This is an open-access article distributed unde the terms of the Creative Commons Attribution License, which permits unrestricted use, distribution, and reproduction in any medium, provided the original author and source are credited. 
of biosafety training programs regarding the appropriate handling of clinical samples and instruments [9].

\section{Methods}

A survey was conducted to know the level of awareness and use of biosafety practices among laboratory personnel in various areas of Karachi. A consent documents were properly signed from each participant, a standard was designed and was distributed among different Private Hospitals and Diagnostic Labs personnel situated in different areas of Karachi including SITE Area, Orangi Town, North Nazimabad, Nazimabad, New Karachi, Sadar, Malir Cant, Gulshaniqbal, Gulistan-johar, Tariq road, Quaid Abad and F.B Area. The survey was designed including basic questions about all the routine laboratory work and practices such as specimen collection, handling and processing, use of PPE, practice on fire extinguishers, availability of SOPs, biological waste categorization, mouth pipetting of samples, dangerous work practices (i.e drinking and eating in laboratories working areas) and proper disposal of biological wastes, sharps and used syringes etc.

- Sampling Plan: Random Sampling was done from employs of the Labs.

- Study Design: The survey was designed on quantitative basis.

- Sample Size: The size of the sample for this survey was 120 with added $10 \%$ to cover the non-respondents; total of 132 respondents based on a previous study [10].

Consent documentation was obtained from supervisors and incharge persons, the technicians, supervisors, assistant technicians, and trainees were interviewed by survey team members and questionnaire was filled out by them. Questionnaires were containing close-end questions.

\section{Results}

This study was conducted in 12 different areas in Karachi city containing hospital-based and individual diagnostic laboratories. The survey was conducted in 3 months duration from May 2017 to July 2017. The total number of contributors was 132, of which 85 were males and 47 were female. Technicians with working knowledge of more than 5 years were about $59 \%$ of total. These personnel were observed and evaluated for biosafety practices in diagnostic labs. The result of the data was very similar among hospital based labs and other labs situated in different areas of Karachi. Therefore the BSL-2 laboratories are of high concern for the improvement of Laboratory workers, their knowledge about biosafety, handling the hazardous and dangerous infectious agents, safe handling of various virulent pathogens etc.

\section{Discussion}

This study was conducted in 12 different areas in Karachi city containing hospital-based and individual diagnostic laboratories. The survey was conducted in 3 months duration from May 2017 to July 2017. The total number of contributors was 132, of which 85 were males and 47 were female. Technicians with working knowledge of more than 5 years were about $59 \%$ of total. These personnel were observed and evaluated for biosafety practices in diagnostic labs. The result of the data was very similar among hospital based labs and other labs situated in different areas of Karachi. Therefore the BSL-2 laboratories are of high concern for the improvement of Laboratory workers, their knowledge about biosafety, handling the hazardous and dangerous infectious agents, safe handling of various virulent pathogens etc.

\section{Personal protective equipment}

All the surveyed labs were BSL-1, 2 and wearing personal protective equipment is a vital requirement for all lab workers. For example, use of gloves has important role to protect personnel from direct contact with infectious and other pathogenic materials. Contaminated gloves performance as a means to spread infection to environmental surfaces it is essential for the personnel to know how to use PPE. In this study, $65 \%$ of the laboratory personnel did not practice any type of PPE; this was more communal in the (44.2\%) than in hospital based labs (20.8\%). Lab coats and gloves both were used by $15.5 \%$ of the personnel, although gloves or a lab coat were used by $8 \%$ and $11 \%$, respectively. These percentage results are very low when to compare with a study performed in Turkish where $87.4 \%$ and $91.3 \%$ of the lab workers used lab coats and gloves respectively [8]. A survey study from Karachi stated that out of 44 clinical laboratories estimated, gloves were used by lab workers in only two (4.5\%) laboratories [9]. Protection of face and eyes from splashes during different lab activities is must, therefore safety glasses and mask must be used, but in this study we find that only $0.5 \%$ of the technicians used safety glasses or other personal protective equipment (Figure 1 and Table 1).

\section{Disinfecting work benches}

In laboratory, a disinfectant should be applied on all the work benches, safety cabinets and other surfaces of the lab before starting work and after work completion. But in this study shows that about $60 \%$ of lab personnel disinfect their working tables, and about $30 \%$ never disinfect their working benches daily. While the remaining

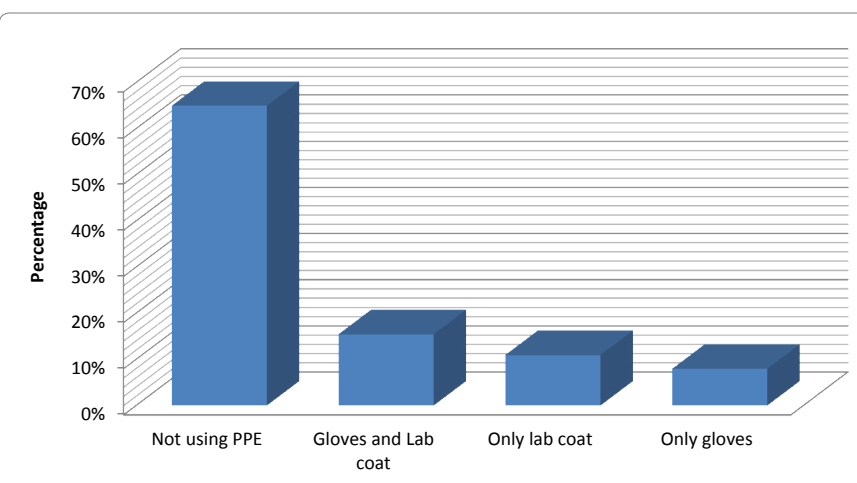

Figure 1: Percentage of laboratory technicians using personal protective equipment during laboratory work.

\begin{tabular}{|c|c|c|c|c|}
\hline \multirow{2}{*}{ S. No: } & \multirow{2}{*}{ Area Name } & $\begin{array}{c}\text { Over all } \\
\text { surveyed labs }\end{array}$ & \multicolumn{2}{|c|}{ Types of Laboratory } \\
\cline { 5 - 5 } & & Hospital Based & $\begin{array}{c}\text { Diagnostic } \\
\text { Labs }\end{array}$ \\
\hline 1 & SITE Area & 8 & 2 & 6 \\
\hline 2 & Orangi Town & 10 & 4 & 6 \\
\hline 3 & North Nazimabad & 18 & 7 & 11 \\
\hline 4 & Nazimabad & 9 & 4 & 5 \\
\hline 5 & New Karachi & 11 & 4 & 7 \\
\hline 6 & Sadar Area & 15 & 5 & 10 \\
\hline 7 & Malir Cant & 12 & 4 & 11 \\
\hline 8 & Gulshan-e lqbal & 17 & 6 & 6 \\
\hline 9 & Gulistan-e Johar & 10 & 4 & 7 \\
\hline 10 & Tariq Road & 6 & 3 & 4 \\
\hline 11 & Quaid Abad & 10 & 3 & $\mathbf{8 4}$ \\
\hline 12 & F.B Area & 6 & 2 & 3 \\
\hline & Total & 132 & 48 & \\
\hline
\end{tabular}

Table 1: List of surveyed laboratories in different areas of Karachi. 
personnel disinfect laboratory surfaces on weekly or monthly basis (Figure 2).

\section{Proper disposal of syringes and sharps}

Recycle of disposable syringes and their inappropriate disposal is a main route of transmission for hepatitis B virus [11], HIV [12], hepatitis C virus [13], abscesses [14], septicaemia [15], malaria [16] and viral hemorrhagic fevers $[17,18]$. In this study, out of the average of $65 \%$, shows that the majority of respondents (35\%) are recapping needles regularly after use, while the others (25\%) recapped them occasionally. To avoid recycle of needles it is recommended that syringes should be cut after use and before disposal. According to a study the proportion of disposable syringe recycle was $24 \%$, and most of the healthcare providers were unaware of the health consequences of recycling disposable syringes [19]. Therefore they must be discarded appropriately to avoid its reuse. The present survey shows that $65 \%$ of the respondents throw sharps and syringes into dustbin without using appropriate disposal procedures. These findings also supported the study done from Karachi which reported that only 35\% of healthcare facilities cut needles prior to disposal [20]. In contrast of this, about $65 \%$ laboratory workers do not place used syringes in isolated sharp containers or discard sharps properly. This ignorance may be one of causes of serious injuries in Karachi (Figure 3).

\section{Mouth pipetting}

Mouth pipetting is considered outdated and is strictly forbidden in clinical laboratories [21]. Though, in this present study approximately $45 \%$ lab workers used mouth pipetting for different purposes. Out of which $21.4 \%$ for blood samples, $15.7 \%$ for chemicals and $7.9 \%$ for preparation of various chemicals dilutions (Figure 4).

\section{Centrifuge machines}

Centrifuge machines are the most common vectors for the dispersal of aerosol and other infectious agents in the laboratory setting as it exert force needed to produce respirable aerosols while spinning at a greater velocity. When these aerosols or air particles are inhaled, it could results a laboratory acquired infection. Therefore, while centrifuging, the tubes should be closed with a suitable cap to avoid biohazards due to inhalation [21]. It is also necessary that centrifuge machine should be properly closed before operating. However, in the present study $33.5 \%$ of hospital based lab technicians and $36.5 \%$ of other lab technicians only occasionally or never close centrifuge machines while centrifugation. But according to an Indian study $63 \%$ of lab workers did not close the lid of centrifuge machine while operating (Figure 5) $[10,22]$.

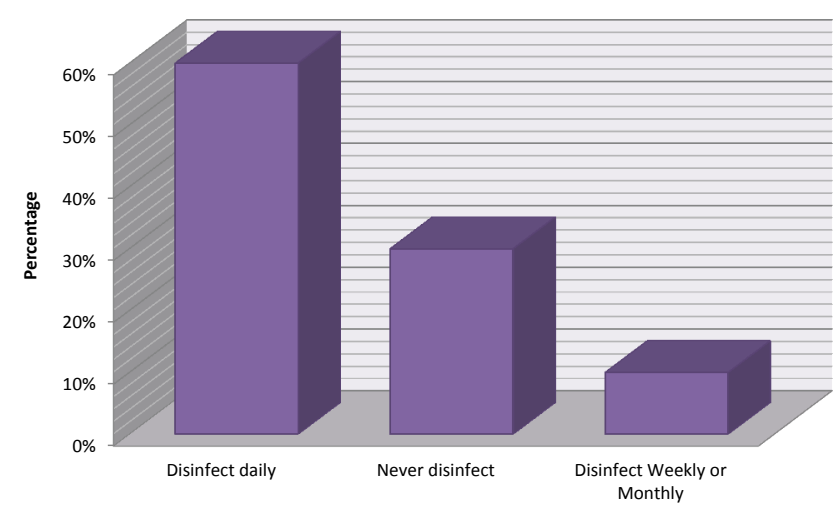

Figure 2: Percentage of the laboratory bench disinfection.

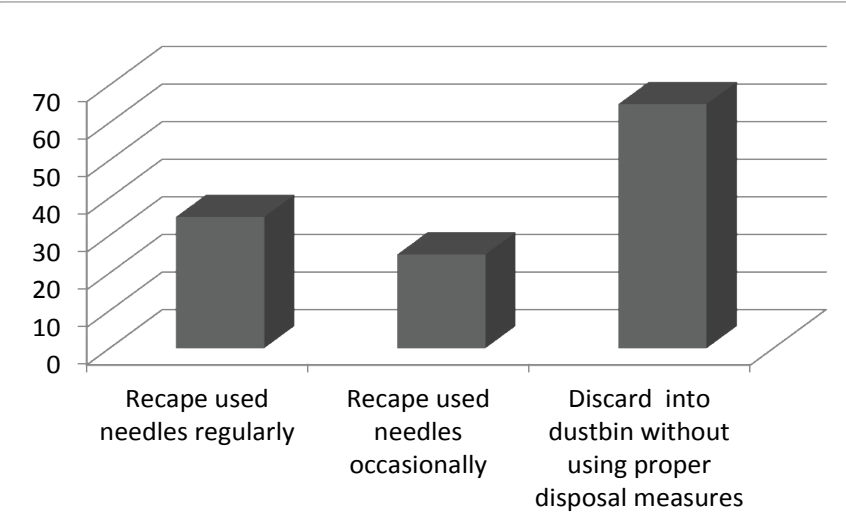

Figure 3: Percentage of technicians reporting the handling and discarding procedures for used syringes and sharps.

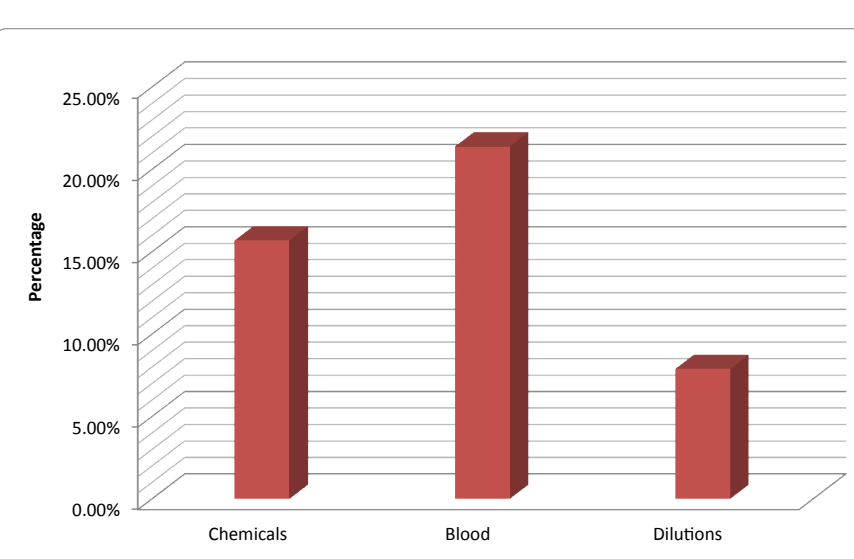

Figure 4: Percentage of technicians performing mouth pipetting for various purposes.

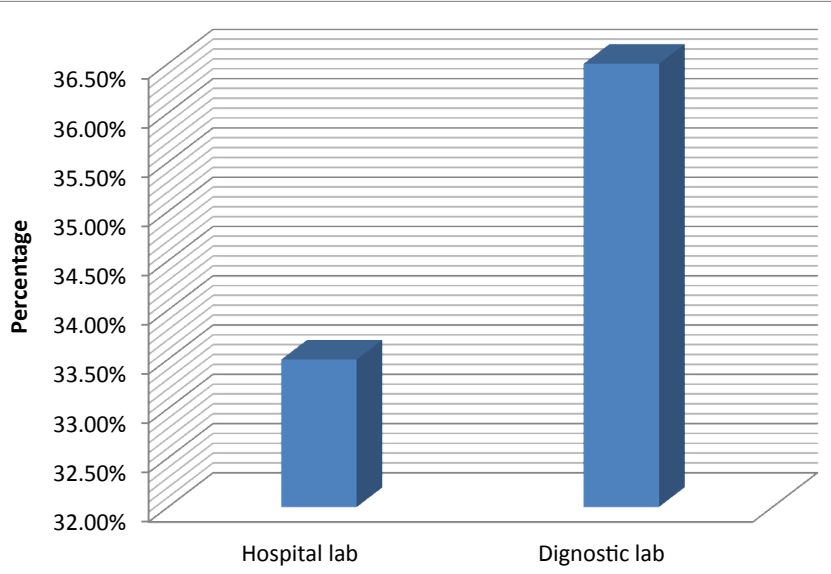

Figure 5: Percentage of hospital based lab technicians and other lab technicians.

\section{Fire extinguishers, biosafety cabinets and a separate place for eating and drinking}

The necessary requirements for BSL-2 are fire extinguishers, biosafety cabinets and a separate place for eating and drinking, this study shows that $58 \%$ of the lab personnel were not properly trained on fire extinguishers. $87 \%$ of labs personnel work without biosafety cabinets due to unavailability biosafety cabinets. Majority (75\%) of the 
personnel were eating and drinking in laboratory areas due to lack of separate places for it (Figure 6).

\section{SOPs, emergency records, and biosafety training}

On average basis, $59.9 \%$ of labs personnel were working without written standard operating procedures (SOPs) and, $72.5 \%$ of labs do not have any maintained accidental records. Similarly, $65 \%$ of respondents were working without any professional training in biosafety (Figure 7).

The results of this present study show that maximum number of the laboratory workers do not have basic awareness associated to biosafety practices. According to another previous study conducted in Karachi, out of 44 laboratories only two to three laboratories practice using gloves during bench work while this study shows that only $15.5 \%$ of the laboratory workers use both gloves and lab coat, while gloves and lab coat were alone used by $8 \%$ and $11 \%$ respectively. Recapping and recycle of syringes is strictly forbidden in BSL-2 labs and all sharps must be properly disposed off after use, in the present study $35 \%$ workers recap syringes on daily basis, $65 \%$ disposed syringes and other sharps without proper measures. Mouth pipetting is also considered outdated and is firmly prohibited in clinical laboratory. However, in this study approximately $45 \%$ lab workers used mouth pipetting for different purposes. Out of which $21.4 \%$ for blood samples, $15.7 \%$ for chemicals and $7.9 \%$ for preparation of various chemicals dilutions.

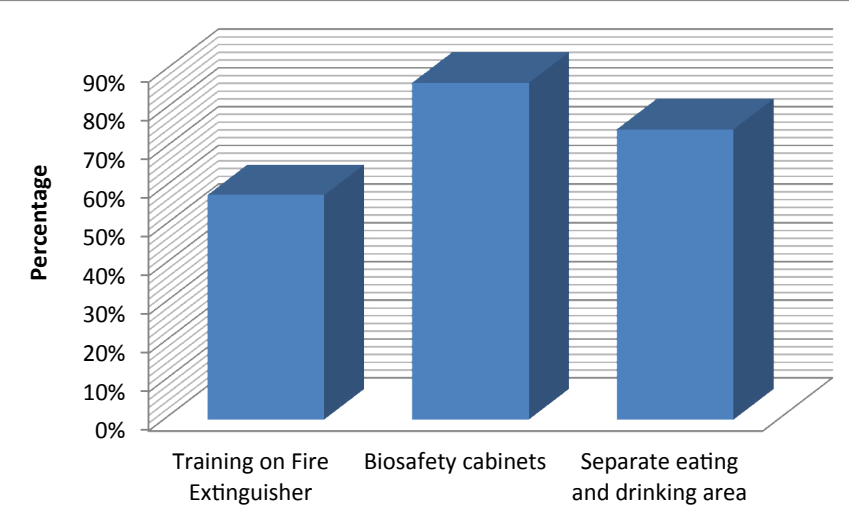

Figure 6: Percentage of biosafety cabinets, fire extinguishers, and a separate place for eating and drinking.

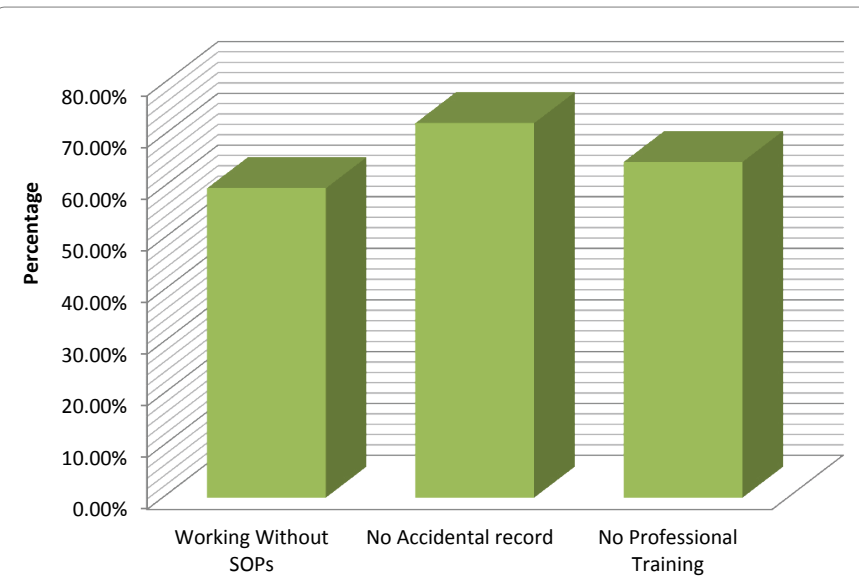

Figure 7: Percentage of biosafety cabinets, fire extinguishers, and a separate place for eating and drinking.
Centrifuge machines are the most common vectors for the dispersal of aerosol and other infectious agents in the laboratory environment as it exert force needed to produce respirable aerosols while spinning at a greater velocity. It is also necessary that centrifuge machine should be properly closed before operating. However, in the present study $33.5 \%$ of hospital based lab technicians and $36.5 \%$ of other lab technicians only occasionally or never close centrifuge machines while centrifugation. Though for BSL-2 laboratory it is necessary to have these facilities like biosafety cabinets, training on fire extinguishers, eye washer, emergency shower, and separate place for eating and drinking, this study shows that $58 \%$ of the lab personnel were not properly trained on fire extinguishers, $87 \%$ of labs personnel work without biosafety cabinets due to unavailability biosafety cabinets. Majority (75\%) of the personnel were eating and drinking in laboratory areas due to lack of separate places for it. Proper SOPs, proper biosafety training and properly maintained accidental record is necessary in every lab, According to another study in 2012; up to $85 \%$ of the workers did not have training courses on biosafety in Sindh, Pakistan. The present study shows that $65 \%$ of the participants in lab did not receive any biosafety courses or training, 59.9\% were working without any SOPs, while $72.5 \%$ labs don not have any maintained accidental record $[23,24]$.

\section{Conclusion}

The results of this survey report confirmed that there is an absence of awareness about good and hygienic lab practices among the lab personnel in different areas of Karachi, Pakistan. Proper institutional based awareness is necessary to address this lack. A biosafety institutional support is required to maintain, control and record the laboratory acquired infection and accidents. In addition, it is needed to organize basic training programs for increasing awareness of basic selfhygienic procedures and biosafety principles in laboratory workers. There should be a biological safety officer, who will be responsible to maintain the biosafety measures within lab and will also oversight the activities like procedures, personnel, storage, equipment, material transfer and transport, proper destruction of biological material etc. There must be efficient implementation of conceptualized strategic framework in all Public and private sectors laboratories to meet the goals and to maintain the biosafety measures properly. Since Pakistan is among developing countries, beside other countries there is a lot of progresses in advanced biological and biomedical researches.

\section{Acknowledgment}

We are grateful to Professor Dr. Shahana Urooj Kazmi, Dean. Faculty of Science Dadabhoy Professor Dr. Rakhshanda Baqai, HOD Clinical Microbiology and Immunology, Institute of Higher Education, Karachi for their support and guidance. We would also like to say thanks to all private-sector hospitals based and diagnostic laboratories for their consent and cooperation to complete this study.

\section{References}

1. U.S. Department of health and human service, center for disease control and prevention, guidelines for safe work practices in human and animal medical diagnostic laboratories, recomm. Biosafety blue ribbon panel Supplement Pp: 7-37.

2. Sewell DL (1995) Laboratory associated infections and biosafety. Clin Microbio Rev 8: 389-405.

3. Saini S, Nagarajan SS (2005) Knowledge, Attitude and Practices of Bio-Medical wastemanagement amongst staff of a tertiary level hospital in New Delhi, India. J Academy of Hospital Administration 17: 1-12

4. Casanova L, Alfano-Sobsey E, Rutala WA, Weber DJ, Sobsey M (2008) Virus transfer from personal protective equipment to health care employee: Emerging Infectious Disease 14:1291-1293. 
Citation: Ahmad S, Ali B, Khan S, Fatima A, Saeed M, et al. (2018) A Survey of Biosafety Practices in Clinical Laboratories Personnel from 12 Selected Areas of Karachi, Pakistan. Health Care Current Reviews 6: 234. doi: 10.4172/2375-4273.1000234

Page 5 of 5

5. Luksamijarulkul P, Kiennukul N, Utrarachkij F, Vatanasomboon P (2010) Current situation of biosafety practices in selected hospital laboratories. Asia $\mathrm{J}$ of Pub Heal 1: 20-22.

6. Nasim S, Shahid A, Mustufa MA, Kazmi SU, Siddiqui TR, et al. (2010) Practice and awareness regarding biosafety measures among lab technicians working in clinical laboratories, Karachi, Pakistan, Applied Biosafety 15: 172-179.

7. Karamat KA, Rahim E, Khattak FH, Mahmood B (2005) Strategic framework for bio-safety and bio-security in public sector hospitals Pakistan. Karachi.

8. Aksoy U, Ozdemir MH, Usluca S, Ergönen AT (2008) A biosafety profile of laboratory workers at three education hospitals in Izmir, Turkey. Mikrobiyoloji Bülteni 42: 469-476.

9. Mujeeb SA, Adil MM, Altaf A, Shah SA, Luby S (2003) Infection control practices in clinical laboratories in Pakistan. Infect Control Hosp Epidemiol 24: 141-142.

10. World health organization (WHO) (1993) Laboratory biosafety manual.

11. Hutin YJF, Harpaz R, Drobeniuc J, Melnic A, Ray C, et al. (1999) Injections given in healthcare settings as a major source of acute hepatitis $B$ in Moldova. Int J Epidemiol 28: 782-786.

12. Hersh BS, Popovici F, Jezek Z, Satten GA, Apetrei RC, et al. (1993) Risk factors for HIV infection among abandoned Romanian children. AIDS 7: 1617-1624.

13. Luby SP, Qamruddin K, Shah AA, Omair A, Pahsa O, et al. (1997) The relationship between therapeutic injections and high prevalence of hepatitis $\mathrm{C}$ infection in Hafizabad, Pakistan. Epidemiol Infect 119: 349-356.

14. Soeters R, Aus C (1989) Hazards of Injectable Therapy. Trop Doct 19: 124-126.

15. Archibald LK, Ramos M, Arduino MJ, Aguero SM, Deseda C, et al. (1998) Enterobacter cloacae and Pseudomonas aeruginosa polymicrobial bloodstream infections traced to extrinsic contamination of a dextrose multidose vial. $\mathrm{J}$ Pediatrics 133: 640-644.

16. Abulrahi HA, Bohlega EA, Fontaine RE, Al-Seghayer SM, AI Ruwais AA (1997) Plasmodium falciparum malaria transmitted in hospital through heparin locks. Lancet 349: 23-25.

17. Fisher-Hoch SP, Tomori O, Nasidi A, Perez-Oronoz GI, Fakile Y, et al. Review of cases of nosocomial Lassa fever in Nigeria: The high price of poor medical practice. BMJ 311: 857-859.

18. Hutin YJF, Hauri AM, Armstrong GL (2003) Use of injections in healthcare settings worldwide 2000: Literature review and regional estimates. BMJ J 327: 1075.

19. Kamal K, Khan A (2003) Re-use of disposable syringes in private health care facilities of Rawalpindi. Pakistan Armed Forces Medical J 53: 234-238.

20. Habibullah S, Afsar S (2007) Waste disposal of government health-care facilities in urban area of Karachi-A KAP survey. Pakistan J Med Resources 46: 1-4.

21. U.S. Department of health and human services, centers for disease contro and prevention, \& national institutes of health. Biosafety in microbiological and biomedical laboratories.

22. Misra UB, Agarwal AK, Parmar NK, Bhalwar R (2001) An epidemiologica study of biohazards in a micro biology laboratory at a large teaching hospital. $J$ Academic Hospital Administration 12: 2.

23. Kermode M, Jolley D, Langkham B, Mathew S, Crofts N (2005) Occupationa exposure to blood and risk of bloodborne virus infection among health care workers in rural north Indian health care settings. Am J Infect Control 33: 34-41.

24. Centers for disease control and prevention (CDC) (2004) Guidance for the selection and use of personal protective equipment (PPE) in healthcare settings. 